\title{
A Perspective from the Neurocritical Care Society and the Society of Critical Care Medicine: Team-Based Care for Neurological Critical Illness
}

\author{
Lewis Kaplan ${ }^{1,2}$, Asma M. Moheet ${ }^{3}$, Sarah L. Livesay4 , J. Javier Provencio ${ }^{5}$, Jose I. Suarez ${ }^{6}$, Mary Kay Bader ${ }^{7}$, \\ Heatherlee Bailey ${ }^{8}$ and Cherylee W. J. Chang ${ }^{9,10^{*}}$
}

(๑) 2020 Springer Science+Business Media, LLC, part of Springer Nature and Neurocritical Care Society

\begin{abstract}
The Neurocritical Care Society and the Society of Critical Care Medicine have worked together to create a perspective regarding the Standards of Neurologic Critical Care Units (Moheet et al. in Neurocrit Care 29:145-160, 2018). The most neurologically ill or injured patients warrant the highest standard of care available; this supports the need for defining and establishing specialized neurological critical care units. Rather than interpreting the Standards as being exclusionary, it is most appropriate to embrace them in the setting of team-based care. Since there are many more patients than there are highly specialized beds, collaborative care and appropriate transfer agreements are essential in promoting excellent patient outcomes. This viewpoint addresses areas of clarification and emphasizes the need for collegiality and partnership in delivering the best specialty critical care to our patients.
\end{abstract}

Keywords: Quality, Quality improvement, Specialty units, Specialty critical care unit, Standards, Collaboration, Neurocritical care, Neurocritical care unit, Intensive care, Intensive care unit, Critical care, Team-based care, Transfer

In 2018, the Neurocritical Care Society (NCS) published a statement outlining Standards for Neurologic Critical Care Units [1]. This publication suggested a framework for different levels of neurologic critical care, and how to best resource the levels of care to improve patient outcomes. Under NCS leadership, a multi-professional writing group of NCS members used an iterative process to create and approve a framework for the Standards. A draft was reviewed by the NCS Quality Committee and NCS Guidelines Committee and posted for comment to the NCS membership. Feedback was formally collated, reviewed and incorporated into the final document

\footnotetext{
*Correspondence: cchang@queens.org

${ }^{9}$ Neuroscience Institute/Neurocritical Care, The Queen's Medical Center Neuroscience Institute, Honolulu, HI 96813, USA

Full list of author information is available at the end of the article
}

that was subsequently approved by the NCS Board of Directors.

The publication generated some concerns in the broader critical care community, and several specific issues were raised by the leadership of the Society of Critical Care Medicine (SCCM) for clarification.

These points included:

1. Table 2 delineating neurocritical care unit (NCCU) recommendations varied from the text regarding physician staffing. This point was addressed in subsequent errata [2].

2. In Table 2 , the " $R$ " could be viewed as required by hospital credentials, thereby ensuring that someone board certified in critical care medicine (CCM) will be unable to care for a neurocritical care (NCC) patient, even a low-risk patient being monitored if 
they overflow into a more general intensive care unit (ICU).

3. The Standards seem to suggest that a CCM physician cannot provide care in an NCCU, yet the premise is that a neurointensivist certified by the United Council of Neurological Subspecialties, a non-ACGME pathway, is perfectly capable of attending in the general ICU. This suggestion seemed surprising in the wake of a recent paper that identifies some key differences in general critical care compared to NCC training [3].

4. Additionally, the Standards seemed to imply that an advanced practice provider (APP) or a second-year trainee could serve as the appropriate designee for patient care, yet a board-certified CCM physician could not.

5. There is a concern that these Standards would prevent trauma surgeons board certified in CCM to attend to those with traumatic brain injury as well as other body cavity injury.

6. Lastly, the Standards also raised issues regarding the suitability and capability of a CCM physician and a neurologist to collaboratively treat patients with neurological conditions if both feel capable of providing care. Issues surrounding an implied need to transfer all patients with neurological conditions to a site with a specifically trained neurointensivist were raised as well.

SCCM leadership brought these issues to the NCS and authors of the Standards for clarification and elaboration. What follows is a collaboratively authored viewpoint including members of both societies to address the issues articulated above. Importantly, this viewpoint underscores the importance of team-based care in providing bedside care as well as in creating standards or guidelines that impact patients, facilities, and members of professional organizations.

The most critically ill or injured patients benefit from team-based care, specifically one that is led by an intensivist. Currently, in the USA, that intensivist may develop from a variety of parent specialties followed by focused training in critical care medicine. With the recent adoption of NCC into the ACGME family of CCM programs, virtually all paths to becoming an intensivist will enjoy the same robust oversight and adherence to standards that support knowledge and care excellence. These different adult pathways-the American Boards of Internal Medicine, Surgery, Anesthesiology, Emergency Medicine, Neurosurgery, and Psychiatry and Neurology-raise a variety of questions for clinicians, administrators, insurers, and patients when evaluating care received in an ICU.
As care complexity increases, technology expands, and healthcare system horizontal integration advances, necessary ICU infrastructure also continues to evolve. These elements do not generally depend on the clinician utilizing them with rare exception (e.g., extracorporeal membrane oxygenation). Instead, they are driven by facility imperatives and patient needs. Accordingly, they require updating in response to relevant changes in standard or emerging care. Since NCCUs are relatively new and driven by the relatively new specialty training pathway of NCC, the NCS sought to articulate the necessary infrastructure to successfully and safely establish and operate an NCCU [1]. This effort leveraged baseline elements necessary for any ICU, but also addressed the clinicians who would staff those ICUs. It is in the latter aspect that clarification is ideal to address elements that may be perceived as controversial.

The concept that the most complex patients should be cared for in a setting that provides comprehensive care is well embraced across multiple specialties for clinical conditions and disease entities including trauma, cardiac or aortic disease, and oncology. In this, neurologic disease is identical. While there is no controversy about that concept, the care of those with less complex processes-which occur in large numbers-may generate controversy. This controversy revolves around whether all patients with a specialty disease process (e.g., neurological injury) must be cared for by a subspecialty-trained intensivist or whether general intensivists in conjunction with non-critical care-trained specialists are able to provide an appropriate level of care. Three points inform this discussion, some of which were housed within the above Standards article.

First, the NCS Standards manuscript describes an aspirational state toward which institutions, administrators, specialty societies, and regulatory agencies may work. In evaluating ICU elements that support NCC, some elements are identified as being recommended, denoted as " $R$ " within the accompanying tables. Therefore, it is important to note that " $R$ " elements should not be interpreted as requirements to be met. Since only the NCS participated in the development of these initial standards, the Society Standards may best be viewed as a platform from which other professional organizations may work with the NCS to refine recommendations. Therefore, the "R" in the NCS Standards paper reflects recommended, not required. As is common with the development of standards, these serve as a framework for care and recommendations for program development. These recommendations should always be vetted within the local environment and contextualized for implementation. Although one may envision such work leading to the " $R$ " for "recommended" evolving to "requirement" much in 
the way that the American College of Surgeons (ACS) Committee on Trauma has articulated requirements that support consensus standards for trauma center verification, this was not the intent of the original publication [4].

Second, there are no prospective, randomized, controlled trials comparing care rendered by a subspecialtytrained intensivist-led ICU team to efforts of a general intensivist working as a team in conjunction with a noncritical care-trained specialist. There are prospectively derived data documenting that admission to an NCCU rather than a non-specialty ICU reduces mortality for patients with intracerebral hemorrhage (ICH) [5] and that the addition of a neurointensivist to a neurologic specialty ICU improves care quality, reduces cost, and enhances outcome [6]. Additionally, there are robust retrospective data showing similar benefit from deploying an NCC service in an institution previously without a subspecialty-led NCC team for the care of patients with $\mathrm{ICH}$, ischemic stroke, traumatic brain injury (TBI), and subarachnoid hemorrhage [6-15]. The most recent data demonstrate benefit both in the USA and internationally [16-20]. The Standards manuscript, similar to the constructs in the ACS Committee on Trauma's Resources for the Optimal Care of the Injured Patient, bases its criteria largely on ICU structure and staffing in the USA, which may embrace a different structure and be underpinned by different resources. Therefore, prevailing high-quality care that drives transfer to a more complex facility when patient needs exceed facility capability should be reinforced and appropriate transfer agreements should be embraced.

Third, as is the case with other critical care subspecialties in the USA and likely across the globe, there is an insufficient number of ICU beds to house all patients with neurologic disease if they were to be transferred from outlying facilities. Similarly, there is an insufficient number of certified neurointensivists to staff neurocritical care ICUs in those facilities to obviate patient transfer. Therefore, it is important to recognize that current care, of necessity, must continue to provide critical care outside of facilities with specialty-focused neurocritical care ICUs. With the exception of complex neurocritically ill patients, for which transfer to a specialty-focused NCCU is recommended, a general intensivist in conjunction with a neurologist and/or neurosurgeon may provide care. Consideration should be given to transfer appropriate patients to higher levels of care.

It is in this space that tele-critical care may be particularly useful as a key mechanism to expand the team's resources. An ideal opportunity for such collaboration may be found in those with TBI as well as multi-system injury, especially in those centers where a neurointensivist is not on staff. Therefore, tele-critical care provides another venue by which to embrace and expand team-based care in both civilian and military medical practice [21].

The issue of competency to deliver care within a specialty-focused ICU offers another opportunity for controversy and clarity. No ICU in the USA requires a resident trainee to demonstrate competency in the subspecialty to provide care. Trainees from multiple parent specialties rotate through medical, surgical, trauma, cardiac surgery, coronary, neuroscience, as well as pediatric ICUs garnering cognitive, technical, and decisional skills. Indeed, in many ICUs, teams of trainees concomitantly flow from multiple parent specialties. The notion that a trainee can develop and demonstrate competency prior to finishing parent specialty training-let alone subspecialty training in a critical care discipline-would be misaligned with every other ICU and their educational programs, all of which are ACGME governed. Trainees work with, but not instead of, attending staff and increasingly commonly work with APP in ICUs as well [22]. The NCC Standards manuscript's intent was not to suggest that a second-year trainee was more competent than a general intensivist, or that a general intensivist was not competent to provide care. It did intend to note that there are unique elements of NCC that may be specifically taught to those who will work as part of the NCCU team, and specifically with a trained neurointensivist. In fact, those elements are readily teachable to all whether they are a trainee, an APP, or a non-neurologic disease-focused general intensivist (if they do not already possess such skills).

The Standards for Neurologic Critical Care Units were put forth to provide a document by which critical care providers can advocate for resources, to organize a framework of neurocritical care delivery, and to act as a catalyst for the iterative process by which outcomes in neurocritically ill patients may be evaluated and enhanced. Therefore, while the neurocritical care Standards manuscript might have been perceived as controversial, we would instead view it as outlining opportunities for collaboration in support of team-based care.

\footnotetext{
Author details

${ }^{1}$ Perelman School of Medicine, University of Pennsylvania, Philadelphia, PA, USA. ${ }^{2}$ Corporal Michael I Crescenz VA Medical Center, Philadelphia, PA, USA. ${ }^{3}$ OhioHealth Riverside Methodist Hospital, Columbus, OH, USA. ${ }^{4}$ Rush University, Chicago, IL, USA. ${ }^{5}$ University of Virginia, Charlottesville, VA, USA. ${ }^{6}$ Johns Hopkins University, Baltimore, MD, USA. ${ }^{7}$ Mission Hospital, Mission Viejo, CA, USA. ${ }^{8}$ Durham VA Medical Center, Durham, NC, USA. ${ }^{9}$ Neuroscience Institute/ Neurocritical Care, The Queen's Medical Center Neuroscience Institute, Honolulu, HI 96813, USA. ${ }^{10}$ John A. Burns School of Medicine, University of Hawaii, Honolulu, HI, USA.
}

Conflict of interest

LK is the president-elect of the Society of Critical Care Medicine (SCCM); AMM is a member of the Neurocritical Care Society (NCS) Board of Directors (BOD) 
and a co-first author of the Standards (1); SL receives personal fees from Stryker NV, Lombardi Hill LLC, and Sarah Livesay LLC and is the NCS secretary, member of the NCS BOD, and a co-first author of the Standards (1); JJP is the Liaison between NCS and SCCM and is a past member of the NCS BOD; JIS is the NCS immediate past president and member of the NCS BOD; MKB is the NCS president; HB is the SCCM president; and CWJC is a SCCM Council member, a NCS past president, ex-officio member of the NCS BOD, and the senior author of the Standards (1).

\section{Publisher's Note}

Springer Nature remains neutral with regard to jurisdictional claims in published maps and institutional affiliations.

Published online: 10 February 2020

\section{References}

1. Moheet AM, Livesay SL, AbdelhakT, et al. Standards for neurologic critical care units: a statement for healthcare professionals from The Neurocritical Care Society. Neurocrit Care. 2018;29:145-60.

2. Moheet AM, Livesay SL, Abdelhak T, et al. Correction to: Standards for neurologic critical care units: a statement for healthcare professions from the Neurocritical Care Society. Neurocrit Care. 2019;31:229.

3. Tisherman SA, Spevetz A, Blosser S, et al. A case for change in adult critical care training for physicians in the United States: a white paper developed by the Critical Care as a Specialty Task Force of the Society of Critical Care Medicine. Crit Care Med. 2018;46:1577-84.

4. American College of Surgeons Committee on Trauma. Resources for optimal care of the injured patient. Chicago: American College of Surgeons; 2014.

5. Diringer MN, Edwards DF. Admission to a neurologic/neurosurgical intensive care unit is associated with reduced mortality rate after intracerebral hemorrhage. Crit Care Med. 2001;29:635-40.

6. Varelas PN, Schultz L, Conti M, Spanaki M, Genarrelli T, Hacein-Bey L. The impact of a neuro-intensivist on patients with stroke admitted to a neurosciences intensive care unit. Neurocrit Care. 2008;9:293-9.

7. Suarez Jl, Zaidat OO, Suri MF, et al. Length of stay and mortality in neurocritically ill patients: impact of a specialized neurocritical care team. Crit Care Med. 2004;32:2311-7.

8. Bershad EM, Feen ES, Hernandez OH, Suri MF, Suarez JI. Impact of a specialized neurointensive care team on outcomes of critically ill acute ischemic stroke patients. Neurocrit Care. 2008;9:287-92.
9. Varelas PN, Conti MM, Spanaki MV, et al. The impact of a neurointensivistled team on a semiclosed neurosciences intensive care unit. Crit Care Med. 2004;32:2191-8.

10. Varelas PN, Eastwood D, Yun HG, et al. Impact of a neurointensivist on outcomes in patients with head trauma treated in a neuroscience intensive care unit. J Neurosurg. 2006;104:713-9.

11. Mirski MA, Chang CWJ, Cowan R. Impact of a neuroscience intensive care unit on neurosurgical patient outcomes and cost of care: evidence-based support for an intensivist-directed specialty ICU model of care. J Neurosurg Anesthesiol. 2001;13:83-92.

12. Samuels O, Webb A, Culler S, Martin K, Barrow D. Impact of a dedicated neurocritical care team in treating patients with aneurysmal subarachnoid hemorrhage. Neurocrit Care. 2011;14:334-40.

13. Josephson SA, Douglas VC, Lawton MT, English JD, Smith WS, Ko NU. Improvement in intensive care unit outcomes in patients with subarachnoid hemorrhage after initiation of neurointensivist co-management. J Neurosurg. 2010;112:626-30.

14. Knopf L, Staff I, Gomes J, McCullough L. Impact of a neurointensivist on outcomes in critically ill stroke patients. Neurocrit Care. 2012;16:63-71.

15. Burns JD, Green DM, Lau H, et al. The effect of a neurocritical care service without a dedicated neuro-ICU on quality of care in intracerebral hemorrhage. Neurocrit Care. 2013;18:305-12.

16. Suarez Jl, Martin RH, Bauza C, et al. Worldwide organization of neurocrtical care: results from the PRINCE Study Part I. Neurocrit Care. 2019. https ://doi.org/10.1007/s12028-019-00750-3.

17. Rao CPV, Suarez J, Martin RH, et al. Global survey of outcomes of neurocritical care patients: analysis of the PRINCE study part 2. Neurocrit Care. 2019. https://doi.org/10.1007/s12028-019-00835-z.

18. Jeong JH, Bang J, Jeong $\mathrm{W}$, et al. A dedicated neurological intensive care unit offers improved outcomes for patients with brain and spine injuries. J Intensive Care Med. 2019:34:104-8.

19. Soliman I, Aletreby WT, Faqihi F, et al. Improved outcomes following the establishment of a Neurocritical Care Unit in Saudi Arabia. Crit Care Res Pract. 2018;2018:2764907.

20. Egawa S, Hifumi T, Kawakita K, et al. Impact of neurointensivist-managed intensive care unit implementation on patient outcomes after aneurysmal subarachnoid hemorrhage. J Crit Care. 2016;32:52-5.

21. Davis K, Perry-Moseanko A, Tadlock MD, Henry N, Pamplin J. Successful implementation of a low-cost tele-critical care solution by the US Navy: initial experience and recommendations. Mil Med. 2017;182:e1702-7.

22. Kleinpell RM, Grabenkort R, Kapu AN, Constantine R, Sicoutris C. Nurse practitioners and physician assistants in acute and critical care: a concise review of the literature and data 2008-2018. Crit Care Med. 2019;47(10):1442-9 